\title{
BMJ Open Developing and testing community- based tuberculosis (TB) screening intervention to increase TB referral, case detection and knowledge among sexual minority people in urban Bangladesh: a mixed-method study protocol
}

Golam Sarwar, ${ }^{1}$ Masud Reza, ${ }^{1}$ Mohammad Niaz Morshed Khan, ${ }^{1}$ Gorkey Gourab, ${ }^{1}$ Mahbubur Rahman, ${ }^{1}$ A K M Masud Rana, ${ }^{1}$ Shaan Muberra Khan, ${ }^{1}$ Samira Dishti Irfan, ${ }^{1}$ Shahriar Ahmed (D) , ${ }^{1}$ Rupali Sisir Banu, ${ }^{2}$ Sayera Banu, ${ }^{1}$ Sharful Islam Khan (iD ${ }^{1}$

To cite: Sarwar G, Reza M, Khan MNM, et al. Developing and testing community-based tuberculosis (TB) screening intervention to increase TB referral, case detection and knowledge among sexual minority people in urban Bangladesh: a mixed-method study protocol. BMJ Open 2020;10:e037371. doi:10.1136/ bmjopen-2020-037371

- Prepublication history for this paper is available online To view these files, please visit the journal online (http://dx.doi. org/10.1136/bmjopen-2020037371).

Received 02 February 2020 Revised 01 August 2020 Accepted 03 August 2020

Check for updates

(C) Author(s) (or their employer(s)) 2020. Re-use permitted under CC BY-NC. No commercial re-use. See rights and permissions. Published by BMJ.

For numbered affiliations see end of article.

Correspondence to Dr Sharful Islam Khan; sharful@icddrb.org

\section{ABSTRACT}

Introduction Although Bangladesh is a country of generalised tuberculosis (TB) epidemic, the HIV prevalence is low among general populations, and $3.9 \%$ among key populations. Despite the high possibility of HIV-TB coinfection, scientifically tested approaches for increasing TB case detection among sexual minority people are yet to be developed and implemented in Bangladesh. Such approaches could foster service delivery linkages between communities and the government health system. Findings of this experimental research are likely to provide new insights for programme managers and policy planners for adopting a similar approach in order to enhance TB referral, thus ultimately increasing TB case detections and reducing the likelihood of TB-related mortalities and morbidities, irrespective of HIV status.

Methods and analysis This operational research will follow a quasi-experimental design, applying both qualitative and quantitative methods, in two dropin centres in three phases. Phase 1 will encompass baseline data collection and development of a community-based TB screening approach. In phase 2, the newly developed intervention will be implemented, followed by end-line data collection in phase 3 . Qualitative data collection will be continued throughout the first and second phases. The baseline and endline data will be compared both in the intervention and comparison areas to measure the impact of the intervention.

Ethics and dissemination Ethical approval was obtained from the Institutional Review Board of International Centre for Diarrhoeal Disease Research, Bangladesh. The findings will be disseminated through diverse scientific forums including peer-reviewed journals, presentation at conferences and among the policy-makers for policy implication. The study started in January 2019 and will continue until June 2020.
Strengths and limitations of this study

The study adopts a mixed-method design, which will validate results from quantitative and qualitative strands in order to explicitly understand the research problem.

- The study will use a community-based approach, thus ensuring the involvement of the sexual minority population at all phases of the study to ensure the internal validity of the findings.

- This study will develop an approach of detecting tuberculosis cases for the first time among sexual minority people through an integrated model, which will harness service delivery linkages with the government health system for achieving sustainability.

- This study will follow a quasi-experimental design rather than a randomised controlled trial.

- The study will be conducted in two service centres only; therefore, the findings generated from this study may not be generalised for the whole sexual minority people; nonetheless, the approach is expected to help in informing the policy makers.

\section{INTRODUCTION}

HIV epidemic poses as an impediment to the effective control of tuberculosis (TB) worldwide. ${ }^{1}$ Clinical evidence has alluded to a causal relationship between TB and HIV. ${ }^{2}$ HIV is considered as the most predominant risk factor for the development of Mycobacterium tuberculosis infection to TB disease. $^{2}{ }^{3}$ Likewise, TB infection accelerates the progression of HIV infection, reduces survival and even predisposes the infected individual to premature death. ${ }^{4} \mathrm{On}$ the global scale, an estimated 10.0 million 
(range 9.0-11.1 million) people were newly diagnosed with TB in 2018, which also constituted to $8.6 \%$ of the People Living with HIV (PLHIV). ${ }^{5}$ An estimated 1.2 million (range, 1.1-1.3 million) deaths due to TB disease occurs across the globe in 2018, in addition to 251000 (range, 223000-281000) TB deaths happened among PLHIV. ${ }^{5}$ This is because PLHIV are 29 (26-31) times more vulnerable to TB than HIV negative individual. ${ }^{6}$ In this context, TB is considered as the leading preventable cause of death especially among PLHIV. ${ }^{7}$

Bangladesh is a country with generalised TB epidemic, though it has a low HIV prevalence of less than $0.01 \%$ among general populations. ${ }^{89}$ However, HIV surveillance findings demonstrated that $3.9 \%$ of the key populations (KPs) at risk of HIV contracted HIV infection. ${ }^{10}$ Between 1999 to 2002, a study conducted by International Centre for Diarrhoeal Disease Research, Bangladesh (icddr,b) among 958 patients to determine HIV prevalence among patients with TB in Dhaka demonstrated an estimated $0.1 \%$ TB/HIV coinfection. ${ }^{11}$ Nonetheless, recent data reported by Government of Bangladesh $(\mathrm{GoB})$ from 2009 to 2014 showed that merely $1 \%$ of the registered TB patients in the country were documented to know their HIV status. ${ }^{12}$ In 2017, the prevalence of HIV among the diagnosed TB patients $(\mathrm{n}=3917)$ was $0.36 \%$ whereas approximately $16 \%$ of the PLHIV $(n=559)$ were tested positive for $\mathrm{TB}$, thus illustrating bidirectional upward trends in TB/HIV coinfection rates over the years. ${ }^{13}$ Moreover, despite of being a country of high TB burden, TB case detection rate (the yearly number of newly notified cases per 100000 population) in Bangladesh is still unsatisfactory as at a $75 \%$ case detection rate as of $2018 .^{5}$

In Bangladesh, TB control interventions are being implemented in healthcare facilities throughout the country, from the central to peripheral levels. HIV prevention programmes are primarily concentrated in 23 priority districts based on concentration of HIV positive cases and KPs. ${ }^{14}$ Thus, there is inadequate linkage between TB and HIV prevention programmes, that engendering low identification of TB cases among KPs. To foster a functional collaboration between TB and HIV programmes, GoB designed a roadmap of collaboration between the National Tuberculosis Control Program (NTP), AIDS/STD Program, key stakeholders (BRAC, icddr,b, Save the Children, etc) and implementing partners (Damien Foundation, CARE,B, etc) since 2009 with an aim to institute programmatic linkages at each level of healthcare, although with unsatisfactory outcomes. ${ }^{12}$

Worldwide, KPs, including sexual minority people (ie, men who have sex with men (MSM), male sex workers (MSW) and transgender women (locally known as hijra)), people who inject drug, female sex workers and PLHIV, are considered vulnerable to TB. ${ }^{15}$ These populations are particularly susceptible to TB infection due to their hidden and hard-to-reach nature along with their unwillingness to visitor lack of access to healthcare facilities like other vulnerable populations. ${ }^{16}{ }^{17}$ In particular, sexual minority people are more vulnerable not only because of their propensity to contract TB/HIV coinfections but also their highly stigmatised nature. ${ }^{18}$ Case notification rates also remain low among these populations due to the implementation of passive case detection of TB control strategy which often exclude marginalised populations, thus leaving them undiagnosed and untreated for TB. ${ }^{19}{ }^{20}$ Experiences from some countries have shown that successful implementation of active case finding strategies, resulted in increased detection of TB case, especially for hidden and/or hard-to-reach populations. ${ }^{21}{ }^{22}$ For example, a recent study conducted in tribal India revealed that active case finding interventions contributed to a $52 \%$ increase in testing rates and $84 \%$ increase in TB case notifications. ${ }^{23}$ Similarly, a recent quasi-experimental study that was conducted in Cambodia showed that the TB case notification rates were significantly higher $(\mathrm{p}<0.05)$ among the intervention group (which adopted active case finding) compared with the control group (which employed passive case finding).$^{24}$ Likewise, a recent cohort study conducted in China documented that early case detection rates significantly increased among the higher risk groups, such as PLHIV $^{25}$.

Several studies have suggested that TB screening should be encompassed in an integrated HIV intervention, while also addressing the other healthcare needs of PLHIV. For example, a community outreach model was adopted in rural Mozambique, where TB, HIV, malaria and nutritional services are packaged in a single service delivery point, which helped improve acceptability and satisfaction. ${ }^{26}$ However, the acceptability of TB screening programmes among MSM has not been a substantial point of focus in research or programme implementation. In fact, many NTP programmes in low/middle-income countries are oblivious to the role that MSM may contribute in TB epidemics. It was evidenced that even MSM, rooted in higher income countries, are at increased risk for TB. ${ }^{27} 28$ The acceptability of TB screening was found to range from $43 \%$ to $91 \%$ in many of the studied countries, although the underlying reasons were not specified. TB screening service acceptability among transgender women is also an understudied area. The reach and recruitment rates of transgender cases were hard to quantify since many of the studies used word of mouth or peer referrals through snowball sampling. ${ }^{29}$

In Bangladesh, $77 \%$ of the transgender women (locally known as hijra) articulated their willingness to provide sputum samples. ${ }^{19}$ According to Mitchell et al, it was inconvenient to reach transgender participants through traditional contact techniques. ${ }^{29}$ Rather, the Center for Disease Control recounted an experience where they attempted to recruit transgender women through exploring their residences, social networks and other social spheres as well as common social spheres. ${ }^{30}$ Transgender women in Bangladesh, indicated that they felt more comfortable visiting a community-based healthcare facility, particularly if they did not disclose their sexual 


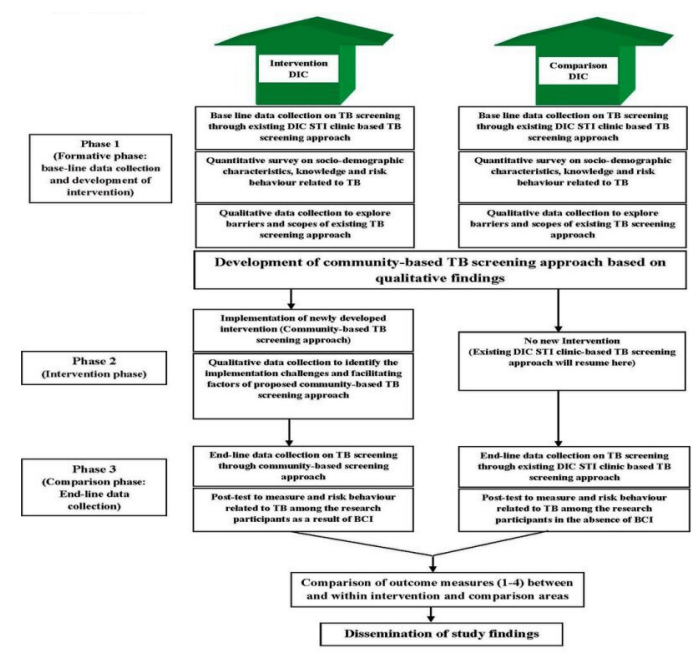

Figure 1 Developing and testing community-based tuberculosis (TB) screening intervention for sexual minority population (men who have sex with men, male sex worker, transgender in Dhaka, Bangladesh Study phases, January 2019-June 2020). BCl, behaviour change intervention; DIC, drop-in centres; STI, sexually transmitted infections.

orientation and if the facilities were catered to sexual minority populations. ${ }^{17}$

icddr,b is currently performing verbal TB screening ('It is a standardised verbal questionnaire for investigating presumptive TB cases on the presence of the following symptoms and signs: cough for more than 2 weeks, haemoptysis, chest pain, breathlessness, evening rise of temperature/night sweating, anorexia, and weight loss/ weakness') for sexual minority people through drop-in centre (DIC)-based TB screening approach. Programmatic evidence showed that this approach is not working efficiently in increasing the number of presumptive TB case identifications and referrals, and active TB case detection. During the preceding 6 -month period (ie, July 2018-December 2018) of inception of the study, only 146 (around 16\%) were screened for TB, using the verbal TB screening form during clinic sessions, yet none were referred as presumptive cases (Presumptive TB case refers to a patient who manifests symptoms or signs which are suggestive of TB (formerly known as a TB suspect); Programme data of icddr,b). National data also demonstrated that additional efforts are required to identify TB cases among highly vulnerable KPs. ${ }^{12}$

A survey conducted in Bangladesh showed the controls had significantly poorer knowledge on mode of transmission, suggestive symptoms and availability of free treatment than the TB cases. ${ }^{31}$ Evidence shows that the knowledge is an essential precursor for successful uptake of TB services and case detection. Knowledge levels and healthcare-seeking behaviours can also contain the spread of TB among vulnerable populations. ${ }^{32}$

Despite high possibility of TB/HIV coinfection, a scientifically tested approach for increasing TB case detection among sexual minority people is yet to be developed and implemented in Bangladesh. Therefore, a community-based TB screening approach will be tested among sexual minority people. Such an approach is expected to encourage sustainable service delivery linkages between community systems and the Government health systems. Findings of this study are likely to provide new insights for programme managers (PMs) and policy planners for developing and implementing a communitybased TB screening approach to enhance TB referral which ultimately increase TB case detection among sexual minority people.

\section{RESEARCH OBJECTIVES \\ Primary objective}

To develop and assess a community-based TB screening approach to enhance $\mathrm{TB}$ referral for increasing case detection among sexual minority people in Dhaka city, Bangladesh.

\section{Secondary objectives}

1. To understand barriers and scopes of the existing TB screening approach for sexual minority people.

2. To measure knowledge of TB among the participants.

3. To develop and implement a community-based TB screening approach.

4. To assess the impact of community-based TB screening approach on TB screening, presumptive TB case referral, TB case detection and knowledge related to TB.

\section{METHODS AND ANALYSIS \\ Research design}

Under the operational research framework, by applying mixed methods (ie, qualitative and quantitative) approach, the study will follow a quasi-experimental design with enlisted sexual minority people in selected DICs. ${ }^{33}$ Both preintervention and postintervention measurements will be facilitated among non-randomly selected control groups. ${ }^{34}$ The participants of intervention site will be compared with the participants of the comparison site. To measure changes between two time points, baseline and end-line in the outcome of interest and between the two groups, an analysis will be conducted.

\section{Research plan}

The study will ensue through the following phases adopted from the operational research framework recommended by WHO and the Global Fund $(\mathrm{GF})^{35}$-figure 1 shows the activities of this study:

\section{Phase 1: formative phase}

1. Qualitative data collection to explore implementation barriers and scopes of existing TB screening approach.

2. Survey on sociodemographic characteristics, knowledge and risk behaviour related to TB among the sexual minority people of the both intervention and comparison catchment areas.

3. Development of community-based TB screening approach. 
4. Baseline data collection on the number of TB screening, referral of presumptive TB cases to directly observed treatment, short-course (DOTS, also known as TB-DOTS) centres and TB case detection within existing DIC STI clinic-based approach of TB screening at both intervention and comparison sites.

Phase 2: intervention phase

1. Implementation of community-based $\mathrm{TB}$ screening intervention.

2. Qualitative data collection to identify implementation facilitators and challenges of community-based TB screening approach.

\section{Phase 3: comparison phase}

1. End-line data collection in both intervention and comparison sites on the number of TB screening, referral of presumptive TB cases to DOTS centres, and TB case detection. The comparison will be done between endline and the baseline data in both intervention and comparison sites.

2. Post test to measure knowledge change as a result of behaviour change intervention (BCI) among sexual minority people in the intervention DIC area and comparison DIC area at the end of the intervention period, of whom the latter will not get BCI. Comparisons will subsequently be drawn within and between these two areas

\section{Development of community-based TB screening model}

A community-based TB screening model will be developed based on the first phase findings. At the beginning of the second phase, researchers will develop a training module for the peer educators (PEs) based on the need assessment, map TB DOTS centres and devise DOTS referral strategies. Sensitisation sessions will be conducted with the DOTS service providers and community leaders. Researchers will train PEs of the intervention area on the TB screening process and then be assigned to serve a separate list of sexual minority people. A work plan will be formulated to screen all sexual minority people at least once during the intervention period. After taking their verbal consents, they will be screened at spots/residences/dera (residence of hijra leader) using a simplified structured verbal TB screening form, while maintaining confidentiality. If the case is negative, post screening information and counselling will be provided and they will be encouraged to seek support if suspected with TB symptoms. If screened positive, they will undergo counselling and be referred to the nearest TB DOTS centre for testing, either through self- or accompanied referral. After the test results are ready, the PE or the sexual minority people themselves will collect the report, and if anyone is tested positive she/he will be linked to nearby TB DOTS centre for treatment and followed up for adherence. PEs will also conduct specialised educational sessions on TB at outreach sites where they will distribute visual materials and discuss $\mathrm{TB}$ risks and routes of transmission.
Study area

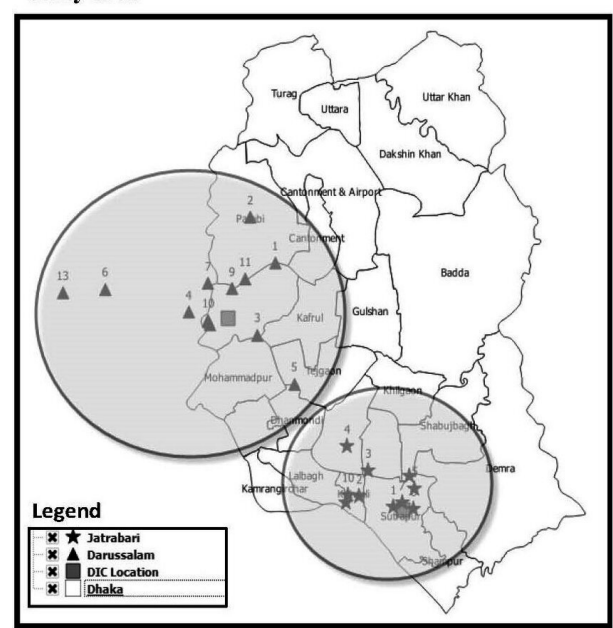

Figure 2 Developing and testing community-based tuberculosis (TB) screening intervention for sexual minority population (men who have sex with men, male sex workers, transgender in Dhaka, Bangladesh Study location/area, January 2019-June 2020). DIC, drop-in centre.

\section{Study location}

This experimental study will be conducted in two DICs (ie, Jatrabari and Darus Salam DIC) in Dhaka city (figure 2). These DICs are operated by a non-government organisation namely Bandhu Social Welfare Society, under the GF project of icddr,b. The intervention site, Jatrabari DIC, is located in the southern part of Dhaka city. Darus Salam DIC, the comparison site, is in the western part of Dhaka city (Mirpur). These DICs were selected purposively due to similar profiles in terms of duration of service delivery, the concentration of HIV positive cases and number of sexual minority people served.

\section{Study population}

The sexual minority people enlisted for HIV prevention services in these two DICs will be selected for this study. Operational definitions for the MSM, MSW and hijra are provided in table 1 which are currently used in the provision of HIV prevention services to sexual minority people. $^{36}$

Table 1 Definitions of the men who have sex with men (MSM), male sex workers (MSW) and hijra

\begin{tabular}{ll}
\hline Population & Operational definitions \\
\hline MSM & $\begin{array}{l}\text { Men who have had sex with men (with } \\
\text { consent) within the last 1 year irrespective of } \\
\text { whether or not they have sex with women } \\
\text { or have a social or personal gay or bisexual } \\
\text { identity, but do not sell sex }\end{array}$ \\
MSW & $\begin{array}{l}\text { Man who sell sex to other men in exchange } \\
\text { of money or gifts in the last } 3 \text { months }\end{array}$ \\
Hijra & $\begin{array}{l}\text { Those who identify themselves as part of a } \\
\text { traditional hijra subculture and who maintain } \\
\text { the guru-chela hijra hierarchy }\end{array}$ \\
\hline
\end{tabular}


For qualitative interview, the following research participants will be recruited purposively in this research:

1. Sexual minority people (MSM, MSW and hijra).

2. DIC staff (ie, DIC managers, medical assistants, outreach supervisors and PEs).

3. Staff of adjacent DOTS centres (ie, physicians, nurses, technicians, etc).

4. Experienced PMs and researchers working with KPs.

5. PMs of NTP.

6. Representative from principal and subrecipients of the GF project.

\section{Inclusion criteria}

1. All sexual minority people aged 18 years or more, and enlisted in the service list of the intervention site (for intervention).

2. According to the sample size, a subset of sexual minority people of both intervention and comparison areas (for baseline and end-line survey).

3. Health service providers and other staff members of the intervention and comparison DICs (for qualitative interview only).

4. Senior management staff members of the PR and SRs, staff of adjacent DOTS centre, experienced PMs of NTP and researchers working with KPs (for qualitative interview only).

5. Those who provide verbal and/or written consent to participate in this study.

\section{Exclusion criteria}

Participants who refuse to participate in this research, and sexual minority people who are not enlisted in the intervention DIC. In addition, minors, below 18 years, will not be included in the study.

\section{Study period}

The study commenced in January 2019 and is anticipated to continue until June 2020. The Gantt chart is given in table 2:

\section{Sample size and sampling}

\section{Quantitative component}

To conduct the baseline survey, in both the intervention and comparison sites, the calculation of sample size was done using the following standard formula ${ }^{37}$ :

$$
n=D \frac{\left\{z_{1-x} \sqrt{2 \bar{p}(1-\bar{p})}+z_{1-\beta} \sqrt{p_{1}\left(1-p_{1}\right)+p_{2}\left(1-p_{2}\right)}\right\}^{2}}{\left(p_{2}-p_{1}\right)^{2}}
$$

In the above formula:

$\mathrm{D}=$ design effect=2 (due to cluster sampling method).

$\mathrm{p}_{1}=$ assumed proportion of the outcome variable at the time of baseline survey.

$\mathrm{p}_{2}=$ the target proportion at the end line, so that ( $\mathrm{p} 2-$ $\mathrm{p} 1$ ) is the magnitude of change that we want to be able to detect

$$
\mathrm{p}(\mathrm{bar})=\left(\mathrm{p}_{1}+\mathrm{p}_{2}\right) / 2
$$

$\mathrm{Z}_{1-\alpha}=$ the $\mathrm{Z}$-score corresponding to desired level of significance $=1.645 .^{37}$

$\mathrm{Z}_{1-\beta}=$ the $\mathrm{Z}$-score corresponding to desired level of power $=0.83 .^{37}$

A literature review was carried out in order to find out estimates of TB knowledge in terms of cause, route of transmission and prevention. The results depicted that the knowledge of cause, routes of transmission and prevention of TB ranged from $2 \%$ to $44 \%, 22 \%$ to $88 \%$ and $14 \%$ to $68 \%$, respectively (table 3 ).

The sample size was calculated in order to detect $20 \%$ changes (1-way change detectable) in the knowledge of TB between two time points with the design effect of 2.0, 95\% CI and $80 \%$ power. Separate sample sizes were calculated for each of the categories and were adjusted for $5 \%$ drop-outs during the interview (table 4). Finally, the largest sample size $(\mathrm{n} \sim 160)$ will be selected to conduct the baseline survey each in the intervention and comparison areas. It is worth mentioning that all the findings were from the general population and no literature was found among the sexual minority people. Thereafter, using programmatic experience among sexual minority people in Bangladesh, suitable values were assumed to calculate sample sizes (table 4).

The baseline survey will be conducted using time location sampling. ${ }^{37}$ The trained interviewers will interview sexual minority people at all congregating areas (spots) at a particular time window, usually between 17:00 and 22:00. After collecting the list of spots and the sexual minority people covered throughout each spot, the total sample size will be proportionately distributed among each spot. In each spot, the respondents will be randomly chosen for interview to collect data on sociodemographic characteristics, knowledge of and risk behaviours pertaining to $\mathrm{TB}$ and so on. Identification numbers (IDs) from the mother list will be used to avoid duplications during the survey. Similar sample size and sampling method will be used to conduct end line survey both in intervention and comparison areas.

\section{Qualitative component}

Non-probabilistic purposeful maximum diversity sampling will be used in the qualitative component. Based on our previous experience of working with sexual minority people, we plan to facilitate in-depth interviews (IDIs) with 15-18 sexual minority people, $10-15$ key informants interviews (KIIs) with service providers. In addition, two focus group discussions (FGDs) will be conducted with sexual minority people and service providers. The sample size may vary depending on the points of data saturation and redundancy. ${ }^{38}$

\section{Data collection method}

Qualitative data collection

Several qualitative data collection approaches will be employed, such as IDIs with the sexual minority people, KIIs with the service providers and FGDs with sexual minority people and service providers. 


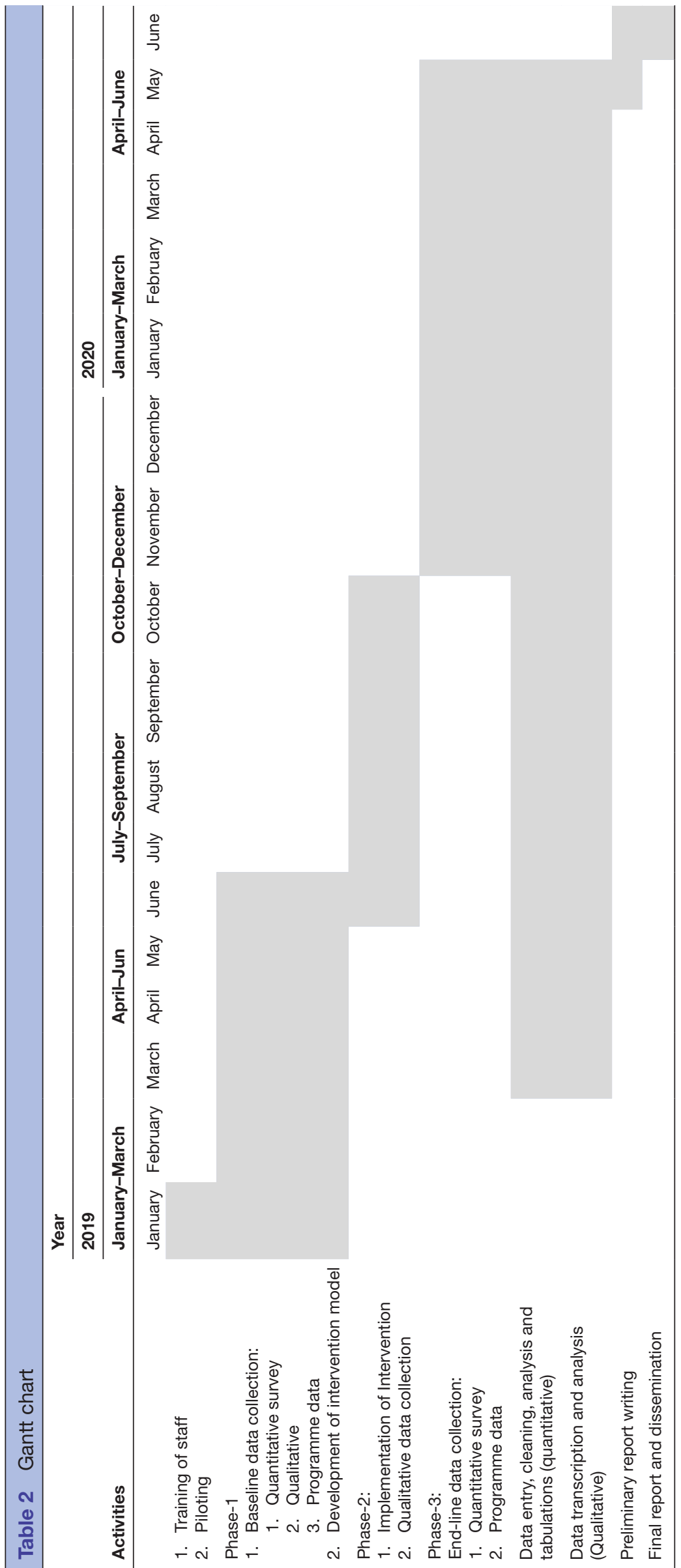


Table 3 Literature review of the knowledge of tuberculosis (TB)

\begin{tabular}{|c|c|c|c|}
\hline Indicators & $\begin{array}{l}\text { Estimates of the } \\
\text { indicators }\end{array}$ & Target group & Source \\
\hline \multicolumn{4}{|l|}{ Knowledge of TB } \\
\hline \multirow{2}{*}{$\begin{array}{l}\text { 1. Cause of TB: percentage of beneficiaries } \\
\text { correctly identify that TB is caused by } \\
\text { bacteria }\end{array}$} & $2 \%$ & General population & Suganthi et al ${ }^{45}$ \\
\hline & $37.7 \%$ & General population & Adane et $a l^{46}$ \\
\hline \multirow{3}{*}{$\begin{array}{l}\text { 2.Route of transmission of TB: percentage } \\
\text { of beneficiaries mentioned that TB is } \\
\text { transmitted by air (by talk/by cough/by } \\
\text { sneezing/by spitting TB germ) }\end{array}$} & $55.5 \%$ & General population & Sreeramareddy et $a l^{48}$ \\
\hline & $56 \%$ & General population & Hossain et $a l^{31}$ \\
\hline & $88.0 \%$ & General population & Adane et $a l^{46}$ \\
\hline $\begin{array}{l}\text { 3. Methods of prevention: percentage of } \\
\text { beneficiaries mentioned that TB can be } \\
\text { prevented by taking BCG vaccination/ } \\
\text { covering mouth and nose when patient with } \\
\text { TB is coughing or sneezing or spitting or } \\
\text { talking }\end{array}$ & $14 \%$ & General population & Anjum et $a l^{47}$ \\
\hline
\end{tabular}

\section{Quantitative data collection}

Survey on sociodemographic characteristics, knowledge of and risk behaviour related to $T B$

Survey on sociodemographic characteristics, knowledge of and risk behaviours related to TB will be conducted among selected numbers of sexual minority people of both intervention and comparison areas during baseline and end-line surveys at an approximate interval of 6 months.

\section{Baseline and end-line programmatic quantitative data collection} At the start and end of the intervention phase of the study, data will be collected from programme report of the GF project of icddr,b on the total number of beneficiaries screened for $\mathrm{TB}$, total number of referred cases for presumptive TB and number of TB case detections within the extant STI clinic-based TB screening approach as well as the new intervention approach at both the intervention and comparison sites.

\section{Data collection tool}

A structured TB screening format will be used for verbal TB screening. The survey will be conducted via a semistructured questionnaire in standard Bengali. This questionnaire will encompass the sociodemographic profile, knowledge of and risk behaviours related to TB. Moreover, open-ended guidelines will be used for qualitative interviews and FGDs. All the guidelines and questionnaires will be field tested. Based on field testing, these data collection tools may need to be refined, where appropriate, in order to facilitate the data collection

Table 4 Proposed sample size to conduct survey with sexual minorities (men who have sex with men, male sex workers, transgender) in Bangladesh (January 2019-June 2020)

\begin{tabular}{|c|c|c|c|c|}
\hline Indicators used in the calculation of sample size & $\begin{array}{l}\text { Suitable values } \\
\text { assumed for the } \\
\text { sexual minority } \\
\text { people }\end{array}$ & $\begin{array}{l}\text { One-way } \\
\text { change } \\
\text { detectable }\end{array}$ & Design effect & $\begin{array}{l}\text { Required } \\
\text { Sample Size* }\end{array}$ \\
\hline $\begin{array}{l}\text { 1. Cause of TB: Percentage of beneficiaries correctly } \\
\text { identify that TB is caused by bacteria }\end{array}$ & $40 \%$ & $20 \%$ & 2.0 & 159 \\
\hline $\begin{array}{l}\text { 3. Methods of prevention: percentage of beneficiaries } \\
\text { mentioned that TB can be prevented by taking BCG } \\
\text { vaccination/covering mouth and nose when patient with } \\
\text { TB is coughing/sneezing/spitting/talking }\end{array}$ & $50 \%$ & $20 \%$ & 2.0 & 152 \\
\hline
\end{tabular}

${ }^{*} 5 \%$ was added in order to adjust drop-out during survey.

TB, tuberculosis. 
process. All qualitative and quantitative interviews will be conducted in standard Bengali.

\section{Outcome variables}

Data to measure outcome variables will be extracted from the ongoing HIV prevention programme from both intervention and comparison areas. These data will be expressed in terms of percentage points to assist statistical analysis. The outcome measures are defined as follows:

1. Number of TB screenings: number of beneficiaries screened for TB at the community, which will be expressed as percentage points in relation to the total number of beneficiaries enlisted in the mother list.

2. Number of presumptive TB case referrals: number of beneficiaries referred to DOTS centres for TB testing, which will be expressed as percentage points in relation to the total number of beneficiaries screened at the community.

3. Number of TB case detections: number of beneficiaries who will be tested positive for $\mathrm{TB}$, which will be expressed as a percentage points in relation to the total number of presumptive cases tested.

Data on knowledge related to TB will be collected from quantitative surveys before intervention (baseline) and after intervention (end line) both in the intervention and comparison areas. This outcome variable will measure the knowledge of TB, as defined below. ${ }^{39}$

1. Cause of TB: correctly able to identify that TB is caused by bacteria.

2. Route of transmission of TB: TB is transmitted by air (by talking/coughing/sneezing/spitting TB germs).

3. Methods of prevention: TB can be prevented by taking BCG vaccination/covering mouth and nose when patient with TB is coughing/sneezing/spitting/talking.

\section{Data analysis}

Quantitative data

Statistical analysis will be conducted using Stata V.13.0 (Stata Corp). Epi Info (V.6.03; Centres for Disease Control and Prevention, Atlanta, Georgia, USA) will be used for data entry. The populations and measurements will be presented through descriptive statistics. Differences in the outcome variables between intervention and comparison groups at the baseline and at end line will be determined by the independent t-test for parametric continuous data, $\chi^{2}$ or Fishers's exact test for categorical data, and Wilcoxon rank-sum test for non-parametric continuous data. Differences in the outcome variables within intervention and comparison areas between two time points will be determined using the paired t-test for parametric continuous data whereas, Wilcoxon signed rank test for non-parametric continuous data and $\chi^{2}$ or Fishers's exact test for categorical data. $\mathrm{P}$ values of $<0.05$ will be considered as a significant level in all comparisons. In addition, 95\% CI will be reported for all quantitative and categorical variables in the study.

\section{Qualitative data}

Data will be collected using digital recorders and systematically stored on the computer on a daily basis for a prolonged timeframe, adhering to the requirement of Institutional Review Board (IRB) and data policy of icddr,b. In addition, data collection and data analysis processes will occur in synergy, as qualitative data collection and analysis is an ongoing and reflexive process, and this process will allow for the identification of saturation points. ${ }^{40}$ All qualitative data will be analysed manually by adopting line-by-line content, contextual and thematic analysis strategies; and coding and recoding data into themes and subthemes. The results will be summarised and presented keeping in mind the local context, and some of the findings will be presented verbatim in order to substantiate or reflect noteworthy ideas and perspectives.

\section{Patient and public involvement}

As mentioned in the 'Study population' section, sexual minority people, enlisted under the intervention and comparison DICs, will be included to explore the research questions and measure outcomes on TB screening, TB presumptive case referral, TB case detection and TB knowledge. While this research did not directly engage them in the design or conceptualisation of the study, researchers planned to involve them throughout the data collection and analysis period. The study participants will be first engaged before the data collection period, when they will be preliminarily oriented about the study objectives and procedure. They will also be involved in facilitating access to and recruiting additional participants, including hidden and hard-to-reach sexual minority people. To design a viable and contextspecific community-based TB screening model and optimise selection of actors (ie, community health workers, existing PE, new peer volunteer as TB screener, etc) for engagement in TB screening, insights will be sought from sexual minority people and service providers via formative research. Member checking sessions will be also conducted to verify correct findings and interpretative analyses. Sexual minority people will be the key recipients of the intervention. Guidance for Reporting Involvement of Patients and Public (GRIPP) 2 reporting checklist for patient and public involvement is available in online supplemental table 1.

\section{Study limitations}

One of the major limitations is unlike randomised controlled trial, the quasi-experimental study design is unable to establish a strong causal association between intervention and outcome due to the lack of randomisation. ${ }^{42}$ However, to overcome this limitation, a similar comparison group will be assigned, which will not receive the intervention. Moreover, qualitative methods will be integrated with quasi-experimental methods to support or refute the findings of the quantitative evaluation and to assess the effectiveness of the intervention. Thus, the causal inference will be strengthened. Another potential 
limitation might be the chance of insufficient control of some confounding factors, which may influence or contaminate the outcome. ${ }^{43}$ This limitation will be mitigated through employing preintervention and postintervention measurement, and statistical analyses that adjust ('control') the confounder(s). In addition, any positive or negative findings that may influence the expected outcome will be documented and reported to describe the context broadly within which generally causal relationships are constructed and deconstructed. Since the study will adopt the non-probabilistic purposive sampling approach because of the stigmatised and hidden nature of the sexual minority people, this may incur selection bias. ${ }^{43}$ Therefore, maximum variation sampling will be attempted to minimise this bias.

\section{ETHICS AND DISSEMINATION}

Participation in the research process will be voluntary. To ensure maximum congeniality of the informants, verbal consent will be attained from sexual minority people during IDIs and FGDs whereas written consent will be obtained from representative of PRs and SRs, service providers from DOTs centres, experienced PMs, and researchers during KIIs and FGDs. Verbal and/or written consent will also be taken from sexual minority people prior to the quantitative survey, as well as the pretest and post-test phases. Experiences of working with sexual minority people suggest that many sexual minority people withhold information about their identities and practices that characterise them as a KP (eg, involvement in sex trade, sexual orientation) in written form in apprehension of identity disclosure. Structural barriers such as criminalisation of same-sex behaviour and socioreligious stigma impede the uptake of written consent from sexual minority people.

Research participants will be briefed about the objectives of the study, the data collection process and topics of discussion. The researchers ascertained the anonymity of the participants' responses. Moreover, no identifying information will appear in the data collection tools. If any research participant declines the recording of his responses, researchers will take written notes.

During intervention, if any participant is tested positive for TB, then she/he will be linked to the adjacent DOTS centre, to ensure treatment and follow-up of the treatment adherence.

Ethical clearance was obtained from icddr,b's IRB which follows international ethical standards to ensure confidentiality, anonymity and informed consent.

Study findings will initially be disseminated at the organisation and then subsequently, among policymakers and relevant stakeholders to inform the policy for implication. In addition, findings will be disseminated to a diverse audience via different scientific forums such as peer-reviewed journals, and presentations at national and international conferences. Further dissemination initiatives include translational and implementation strategies such as the impact of findings in the national policy.

Author affiliations

${ }^{1}$ Infectious Diseases Division, International Centre for Diarrhoeal Disease Research Bangladesh, Dhaka, Bangladesh

${ }^{2}$ National Tuberculosis Control Program, Directorate General of Health Services, Govt. Of the People's Republic of Bangladesh, Dhaka, Bangladesh

Twitter Shahriar Ahmed @ShahriarSajib

Acknowledgements The protocol for this research received funding from The Global Fund to Fight AIDS, Tuberculosis and Malaria (The Global Fund). icddr,b is also obliged for the contribution of The Global Fund to its research efforts. icddr,b is also grateful to the Governments of Bangladesh, Canada, Sweden and the UK for providing core/unrestricted support.

Contributors GS drafted the manuscript and revised it with inputs from all coauthors. SIK is the senior and corresponding author of this manuscript and was responsible for the overall supervision of information summarisation, exchange and management, analysis of information, and drafting of the manuscript. MR and MNMK also supervised overall information extraction and assisted in the drafting of the manuscript. GG also managed information extraction and helped in writing the manuscript. SMK, SDI and MR reviewed the manuscript and provided valuable suggestions for the overall improvement of the manuscript. SB, RSB, SA and AKMMR have read, review and approved the final manuscript.

Funding This study was supported by The Global Fund to Fight AIDS, Tuberculosis and Malaria (The Global Fund). The grant number is GR-01603.

Map disclaimer The depiction of boundaries on this map does not imply the expression of any opinion whatsoever on the part of BMJ (or any member of its group) concerning the legal status of any country, territory, jurisdiction or area or of its authorities. This map is provided without any warranty of any kind, either express or implied.

Competing interests None declared.

Patient consent for publication Not required.

Provenance and peer review Not commissioned; externally peer reviewed.

Open access This is an open access article distributed in accordance with the Creative Commons Attribution Non Commercial (CC BY-NC 4.0) license, which permits others to distribute, remix, adapt, build upon this work non-commercially, and license their derivative works on different terms, provided the original work is properly cited, appropriate credit is given, any changes made indicated, and the use is non-commercial. See: http://creativecommons.org/licenses/by-nc/4.0/.

\section{ORCID iDs}

Shahriar Ahmed http://orcid.org/0000-0002-8833-4130

Sharful Islam Khan http://orcid.org/0000-0002-7319-1333

\section{REFERENCES}

1 Kwan CK, Ernst JD. Hiv and tuberculosis: a deadly human syndemic. Clin Microbiol Rev 2011;24:351-76.

2 Narain JP, Lo Y-R. Epidemiology of HIV-TB in Asia. Indian J Med Res 2004;120:277.

3 Getahun H, Gunneberg C, Granich R, et al. Hiv infection-associated tuberculosis: the epidemiology and the response. Clin Infect Dis 2010;50 Suppl 3:S201-7.

4 Whalen C, Horsburgh CR, Hom D, et al. Accelerated course of human immunodeficiency virus infection after tuberculosis. Am J Respir Crit Care Med 1995;151:129-35.

5 World Health Organization. Global tuberculosis report 2019, 2019.

6 World Health Organization. Secondary HIV associated tuberculosis: Challenges and key issues. In: Hiv associated tuberculosis: challenges and key issues, 2014. www.who.int/tb/challenges/hiv/ tbhiv_factsheet_2014.pdf.

7 Suthar AB, Lawn SD, del Amo J, et al. Antiretroviral therapy for prevention of tuberculosis in adults with HIV: a systematic review and meta-analysis. PLoS Med 2012;9:e1001270.

8 NTP. National guidelines on TB/HIV management and program collaboration and implementation manual. 2 edn, 2016.

9 ASP. Annual report 2018, 2019. 
10 IEDCR i. Behavioural and serological surveillance amongst key population at risk of HIV in selected Arear of Bangladesh, 2016. Dhaka, Bangladesh: IEDCR and icddr,b, 2017: 133.

11 Alam MS, Sarker MS, Mahmud AM, et al. Low HIV infection rates among tuberculosis patients in Dhaka, Bangladesh. Int J STD AIDS 2005;16:86-8.

12 Government of Bangladesh. National guidelines on TB/HIV management and program collaboration \& implementation manual. Health Mo, 2016.

13 National Tuberculosis Control Programme (NTP). Annual Report. In: Tuberculosis control in Bangladesh. 27, 2018.

14 NASP and UNAIDS. Investment case for fast track strategies: prioritizing investment options in HIV response in Bangladesh to end AIDS by 2030: national AIDS/STD program NASP, 2016.

15 UNAIDS. Unaids data, 2019.

16 Hayward S, Harding RM, McShane $\mathrm{H}$, et al. Factors influencing the higher incidence of tuberculosis among migrants and ethnic minorities in the UK. F1000Res 2018;7:461.

17 Gourab G, Khan MNM, Hasan AMR, et al. The willingness to receive sexually transmitted infection services from public healthcare facilities among key populations at risk for human immunodeficiency virus infection in Bangladesh: a qualitative study. PLoS One 2019;14:e0221637.

18 Mitchell EM, Golub J, Portocarrero A. Acceptability of TB screening among at-risk and vulnerable groups. Geneva: World Health Organization, 2011.

19 McDowell M, Hossain M, Rahman N, et al. Expanding tuberculosis case notification among marginalized groups in Bangladesh through peer sputum collection. Public Health Action 2015;5:119-21.

20 Hossain S, Zaman K, Quaiyum A, et al. Care seeking in tuberculosis: results from a countrywide cluster randomised survey in Bangladesh. BMJ Open 2014;4:e004766.

21 Fatima R, Qadeer E, Enarson DA, et al. Success of active tuberculosis case detection among high-risk groups in urban slums in Pakistan. Int J Tuberc Lung Dis 2014;18:1099-104.

22 Eang MT, Satha P, Yadav RP, et al. Early detection of tuberculosis through community-based active case finding in Cambodia. BMC Public Health 2012;12:469.

23 Vyas A, Creswell J, Codlin AJ, et al. Community-based active casefinding to reach the most vulnerable: tuberculosis in tribal areas of India. Int J Tuberc Lung Dis 2019;23:750-5.

24 Morishita F, Eang MT, Nishikiori N, et al. Increased case notification through active case finding of tuberculosis among household and neighbourhood contacts in Cambodia. PLoS One 2016;11:e0150405.

25 Chen J-O, Qiu Y-B, Rueda ZV, et al. Role of community-based active case finding in screening tuberculosis in Yunnan Province of China. Infect Dis Poverty 2019;8:92.

26 Simon S, Chu K, Frieden M, et al. An integrated approach of community health worker support for HIV/AIDS and TB care in Angónia district, Mozambique. BMC Int Health Hum Rights 2009;9:13.

27 Zolopa AR, Hahn JA, Gorter R, et al. Hiv and tuberculosis infection in San Francisco's homeless adults. prevalence and risk factors in a representative sample. JAMA 1994;272:455-61.

28 Sterling TR, Thompson D, Stanley RL, et al. A multi-state outbreak of tuberculosis among members of a highly mobile social network: implications for tuberculosis elimination. Int $J$ Tuberc Lung Dis 2000:4:1066-73.

29 Mitchell E, Shapiro A, Golub J, et al. A systematic qualitative/ quantitative literature metasynthesis. In: Acceptability of TB screening among at-risk and vulnerable groups. WHO, 2013. http:// www who int/tb/tbscreening/en
30 Miller WM, Miller WC, Barrington C, et al. The where and how for reaching transgender women and men who have sex with men with HIV prevention services in Guatemala. AIDS Behav 2017;21:3279-86

31 Hossain S, Zaman K, Quaiyum A, et al. Factors associated with poor knowledge among adults on tuberculosis in Bangladesh: results from a nationwide survey. J Health Popul Nutr 2015;34:2.

32 Samal J. Perception and knowledge of tuberculosis and its services among slum dwellers in Chhattisgarh. Indian J Respir Care 2017;6:828-31.

33 White H, Sabarwal S. Quasi- Experimental design and methods. In: Methodological Briefs:Impact Evaluation, 2014: 8.

34 Harris AD, McGregor JC, Perencevich EN, et al. The use and interpretation of quasi-experimental studies in medical informatics. $J$ Am Med Inform Assoc 2006;13:16-23.

35 World Health Organization and The Global Fund. Guide to operational research in programmes supported by the global fund. In: Secondary guide to operational research in programmes supported by the global fund, 2008. http://www.who.int/hiv/pub/ operational/or_guide_gf.pdf

36 NASP Save the children. Mapping study and size estimation of key populations in Bangladesh for HIV programs 2015-2016, 2016.

37 Amon J, Brown T, Hogle J, et al. Behavioral surveillance surveys Bss. In: Guidelines for repeated behavioral surveys in populations at risk of HIV, 2000.

38 Baker SE, Edwards R, Doidge M. How many qualitative interviews is enough?: expert voices and early career reflections on sampling and cases in qualitative research, 2012

39 Hassan AO, Olukolade R, Ogbuji QC, et al. Knowledge about tuberculosis: a precursor to effective TB Control-Findings from a follow-up national KAP study on tuberculosis among Nigerians. Tuberc Res Treat 2017;2017:1-8.

40 Ezzy D. Qualitative analysis: practice and innovation 2002. London: Routledge Google Scholar, 2002.

41 Mauthner NS, Doucet A. Reflexive accounts and accounts of reflexivity in qualitative data analysis. Sociology 2003;37:413-31.

42 Schweizer ML, Braun BI, Milstone AM. Research methods in healthcare epidemiology and antimicrobial StewardshipQuasi-Experimental designs. Infect Control Hosp Epidemiol 2016;37:1135-40.

43 Harris AD, Bradham DD, Baumgarten $\mathrm{M}$, et al. The use and interpretation of quasi-experimental studies in infectious diseases. Clin Infect Dis 2004;38:1586-91.

44 Wandwalo ER, Mørkve O. Knowledge of disease and treatment among tuberculosis patients in Mwanza, Tanzania. Int J Tuberc Lung Dis 2000;4:1041-6.

45 Suganthi P, Chadha VK, Ahmed J, et al. Health seeking and knowledge about tuberculosis among persons with pulmonary symptoms and tuberculosis cases in Bangalore slums. Int J Tuberc Lung Dis 2008;12:1268-73.

46 Adane K, Spigt M, Johanna L, et al. Tuberculosis knowledge, attitudes, and practices among Northern Ethiopian prisoners: implications for TB control efforts. PLoS One 2017;12:e0174692.

47 Anjum A, Daud S, Mukhtar F. Tuberculosis. Prof med j 2009;16:61-6.

48 Sreeramareddy CT, Harsha Kumar HN, Arokiasamy JT. Prevalence of self-reported tuberculosis, knowledge about tuberculosis transmission and its determinants among adults in India: results from a nation-wide cross-sectional household survey. BMC Infect Dis 2013;13:16

49 Solliman M, Saleem F, Hassali MA. Assessment of knowledge towards tuberculosis among general population in North East Libya. J Appl Pharm Sci 2012;2:24-30. 